augelite. Inasmuch as these omissions and other mistakes are few and far between, they have no real influence on the value of the tables, and everyone working, not only with minerals but also with inorganic compounds, will find it of great use to have this new edition of Strunz's book on his bookshelf.

F. E. WIOKMaN

Department of Mineralogy

Swedish Museum of Natural History

Stockholm 50, Sweden

On the Systems formed by Points Regularly Distributed on a Plane or in Space. By M. A. BravaIs, translated by Amos J. Shaler from Journal de l'École Polytechnique (1850), 19, 1, being Memoir No. 1 of the Crystallographic Society of America: Obtainable through the SecretaryTreasurer, Dr W. Parrish, Philips Laboratories, Inc., Irvington-on-Hudson, New York, U.S.A. Pp. viii+113, with 41 figs. 1949. Price $\$ 3 \cdot 90$.

This first 'memoir' of the Crystallographic Society of America is a very suitable and dignified memorial not only to the great mason who laid the foundations of structural crystallography, but also to the editing Society which has meanwhile merged its activities with those of the former ASXRED by forming the American Crystallographic Association. The translation of Bravais's paper is well done and enables the reader to follow in English the clear and meticulous arguments, which are given more geometrico in all detail. Those who have been introduced to lattices and space groups by modern direct vectorial and grouptheoretical methods will be interested to see how all the basic facts were found and critically discussed before the formalisms for condensing the proofs had been developed.

In the last chapter, on Polar Lattices, most of the properties of the reciprocal lattice are to be found, except the one which really means reciprocity, namely, that the scalar product of vectors taken from the two lattices be dimensionless. This idea is contained in Gibbs's introduction of reciprocal vector sets; it is an important property because it is closely tied up with the ideas of co-and contra-variance of Fourier transformation, and of the duality between co-ordinate and momentum space. In defining the vector in the polar lattice as dimensionally equal to that of the crystal lattice, Bravais missed a point. No wonder though, because at the time he wrote he must have disliked the idea-if it occurred to him-that a length in the polar lattice should really represent a reciprocal length in space.

Polytechnic Institute of Brooklyn, P. P. Ewald Broolklyn 2, N.Y., U.S.A.
An Introduction to Luminescence of Solids. By H. W. Leverenz. Pp. xv +569, with 143 figs., 23 tables and 1 chart. New York: Wiley; London: Chapman and Hall. 1950. Price \$12;96s.

The great importance that luminescent systems have gained in engineering during the last decades has caused a considerable extension of research in this field. Dr Leverenz has taken a very active part in these investigations, and in this book he gives us his views about both the chemistry and the physics of luminophors. Various new results obtained by the author and his collaborators are mentioned, but results of other investigators are also extensively discussed. Readers of this journal will particularly appreciate that much attention is paid to the chemical build-up of luminescent systems, and the close relations which exist between the optical properties and the crystal structures. As luminescence usually originates in centres with limited extension, the information obtained from it mainly concerns these centres. Only in a few cases, however, is this information sufficiently precise to enable us to draw definite conclusions regarding the atomic configurations; in most cases such conclusions must be based on a complex of by-phenomena.

In this connexion it may be questioned whether the evidence in favour of the view that in the sulphides and selenides the centres consist of activator atoms at interstitial sites is sufficiently strong to accept it as a basis of a classification, as the author does. The acceptance of this view leads to a number of statements regarding ' $i$-centre phosphors' which actually hold only for sulphide phosphors-independent of whether their centres are of the interstitial or of the substitutional type.

A generalization of a similar kind concerns substitutional centres. As it is probable that manganese is often present at normal lattice sites, while on the other hand the luminescence of manganese takes place between levels of the manganese ion ('originative activator') it is stated that substitutional activators are usually of the originative type, a statement which is certainly not generally true (SrS-Eu !). This is regrettable, since these rules were obviously intended to bring order to the immense amount of experimental material. In a book like this, in which the author does not hesitate to speak freely, our opinion is bound to differ from his concerning a number of points. This, however, does not diminish the merits of the book. It gives a broad survey of the field of solid luminescent materials, it contains some very instructive figures, a number of extensive tables, and finally an impressive list of references. It will therefore certainly be of great value both to workers in the field and to all interested in the application of phosphors.

Philips ,iesearch Laboratory

Eindhoven, Holland

\title{
Books Received
}

The undermentioned works have been received by the Editors. Mention here does not preclude review at a later date.

Der Ultraschall und seine Anwendung in Wissenschaft und Technik. By I. Bergmann. Pp. xi + 748, with 460 figs. and 83 tables. Zürich: S. Hirzel Verlag. 1949. Price 50 Swiss francs.
X-ray Studies on Polymorphism. By T. Iтo. Pp. 236, with 100 figs. Tokyo: Maruzen Co. Ltd. 1950. Price $\$ 10 \cdot 00$. 